
FOTOESTABILIDADE DE UM DERIVADO AZALACTÔNICO ENCAPSULADO EM CICLODEXTRINA EM SOLUÇÃO AQUOSA.

\title{
STUDY OF THE PHOTOPHYSICAL BEHAVIOR AND THE PHOTOSTABILITY OF AN AZLACTONE DERIVATIVE ENCAPSULATED IN CYCLODEXTRIN.
}

\author{
KRAUSER, Maike de Oliveira; LAHOUD, Marcelo Galindo; ROSA*, Mauricio Ferreira \\ Universidade Estadual do Oeste do Paraná, Departamento de Química, Rua da Faculdade 645, Jd. Sta. Maria, \\ cep 85903-000, Toledo-PR, Brasil. (fone: +55 45 33797074; fax: +55 45 33797001) \\ ${ }^{*}$ Autor correspondente \\ e-mail:mauricio_rosa@ymail.com
}

Received 07 October 2014; received in revised form 03 February 2015; accepted 04 February 2015

\begin{abstract}
RESUMO
As azalactonas são compostos heterocíclicos bem conhecidos, sendo importantes intermediários na síntese de aminoácidos quirais. Mesmo apresentando altas intensidades de emissão de fluorescência, muito poucos trabalhos foram encontrados na literatura acerca das suas propriedades fotofísicas e fotoquímicas. Devido à baixa solubilidade em solução aquosa realizou-se o estudo fotofísico e fotoquímico destes compostos em solução hidroalcoólica e aquosa na presença de ciclodextrinas, uma vez que, segundo dados da literatura, o encapsulamento pode aumentar a estabilidade da molécula encapsulada assim como modificar suas propriedades fotofísicas. Os resultados mostraram que ocorre encapsulamento da azalactona na cavidade da $\beta$-ciclodextrina ( $b-C D$ ) em meio hidroalcoólico devido ao aumento a intensidade de absorção, entretanto observa-se a diminuição da fotoestabilidade do derivado azalactônico em meio hidroalcoólico.
\end{abstract}

Palavras-chave: oxazolonas, fotofísica, ciclodextrina, fotoestabilidade

\begin{abstract}
The azlactones are well known compounds and are important intermediates in the synthesis of chiral heterocyclic amino acids. Even with high intensities of fluorescence emission, very few studies were found in literature about their photophysical and photochemical properties. Due to the low solubility in aqueous solution, we carried out the photochemical and photophysical study of these compounds in aqueous and hydroalcoholic solution in the presence of cyclodextrins since, according to literature data, the encapsulation can enhance the stability of the encapsulated molecule as well as modify its photophysical properties. The results showed increase of absorption intensity of azlactone after encapsulation in the $\beta$-cyclodextrin (b-CD) cavity in aqueousalcoholic medium, however it is observed the decrease in the photostability azlactone derivative in aqueousalcoholic medium.
\end{abstract}

Keywords: oxazolones, photophysics, cyclodextrin, photostability

PERIÓDICO TCHÊ QUÍMICA • www.periodico.tchequimica.com • Vol. 12 N. 23.

- ISSN 1806-0374 (impresso) • ISSN 1806-9827 (CD-ROM) • ISSN 2179-0302 (meio eletrônico)

(C) 2015. Porto Alegre, RS. Brasil 


\section{INTRODUÇÃO}

As azalactonas, atualmente denominadas oxazolonas, são heterociclos de 5 membros que possuem em seu anel um átomo de oxigênio, um de nitrogênio e ainda uma carbonila exocíclica (Figura 1).

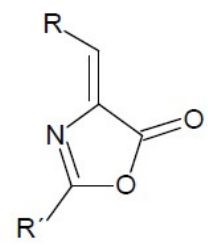

Figura 1 - Fórmula estrutural da azalactonas.

As azalactonas foram primeiramente sintetizadas por Plöchl (1884) pela condensação do ácido hipúrico ( $N$-benzoilglicina) com aldeído aromático em solução de anidrido acético e acetato de sódio, como mostrado no Esquema 1.

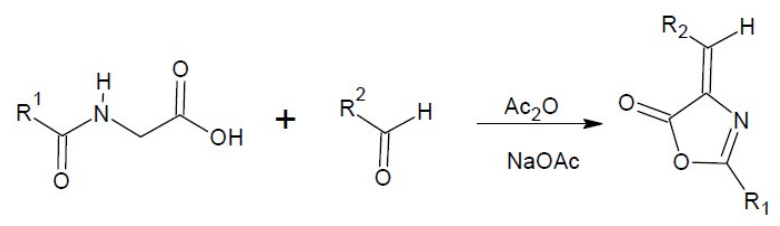

Esquema 1 - Reação descrita por Plöchl

Posteriormente Erlenmeyer (1892) reestudou esta reação propondo a correta estrutura do produto, assim como o emprego deste na síntese de derivados de aminoácidos. Desde então esta reação passou a ser denominada como "Síntese de PlöchlErlenmeyer".

A utilização dos derivados azalactônicos na química é bem diversa. Fisk et al. (2007) apresentaram uma abrangente revisão do emprego desta classe de compostos como intermediários na síntese de uma série de outros compostos de interesse biológico.

Uma importante característica desta família de compostos é alta intensidade de emissão de fluorescência, sendo encontrado na literatura a utilização de derivados azalactônicos como sensores fluorescentes de glicose (ERTEKIN et al. 2005), indicadores fluorescentes de $\mathrm{pH}$ em filmes poliméricos (ERTEKIN et al. 2000) ou em matrizes sol-gel (ERTEKIN et al. 2003), entre outras.

Ozturk; Alp e Ertekin (2005) utilizaram o derivado azalactônico 4-(2-furilmetileno)-2-fenil5-oxazolona suportado em uma matriz polimérica de PVC como sensor fluorimétrico de $\mathrm{Fe}^{3+}$. Os autores observaram a supressão da fluorescência em função do aumento da concentração de $\mathrm{Fe} 3+$ na faixa de $6 \times 10^{-6}$ a $6 \times 10^{-4} \mathrm{~mol} \mathrm{~L}^{-1}$.

Ozturk; Alp; Timur (2008) investigaram a utilização de derivados azalactônicos fixados em matrizes poliméricas de PVC como sensores fluorescentes de acetilcolina e inibidores da acetilcolinesterase. Foi observado que quando expostos a concentrações crescentes de donepezil, um inibidor da acetilcolinesterase, ocorre a supressão de fluorescência do sensor fluorimétrico na faixa linear de $7,99 \times 10^{-7}$ a $443,86 \times 10^{-7} \mathrm{~mol} \mathrm{~L}^{-1}$ para o CPO-I, $7,99 \times 10^{-7}$ a $412,43 \times 10^{-7} \mathrm{~mol} \mathrm{~L}^{-1}$ para CPO-II e 7,99 a $317,88 \times 10^{-7} \mathrm{~mol} \mathrm{~L}^{-1}$ para CPO-III (Figura 2).

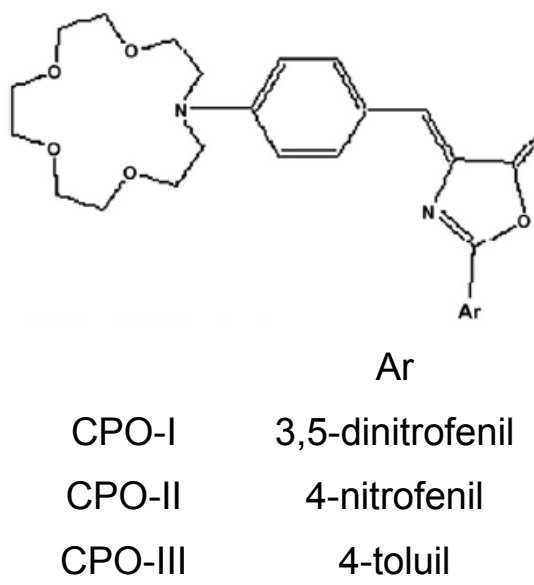

Figura 2 - Estruturas dos derivados 2-(aril)-4-[4(1,4,7,10-tetraoxa-13-azaciclopentadecil)benzilideno]5-oxazolona. (Fonte: Ozturk; Alp; Timur 2008)

Kóczán et al. (2001) utilizaram derivados azalactônicos como fluoróforos no reconhecimento de peptídeos, aminoácidos e aminas. Este reconhecimento ocorre pela alteração da estrutura das moléculas de um derivado de aminoácido através da reação com um derivado azalactônico, conseqüentemente alterando tanto a fluorescência como a absorção do composto, ocorrendo um 
deslocamento batocrômico e ou redução da emissão/absorção do derivado azalactônico.

Foram observadas propriedades
ópticas não-lineares de derivados
azalactônicos em solução (KITAZAWA et al., 1995) ou em estruturas poliméricas (SMOKAL et al., 2007). Em óptica não-linear alguns derivados azalactônicos apresentam a geração de harmônicas, absorvendo o fóton em um determinado comprimento de onda e emitindo o fóton em um múltiplo da onda absorvida. Segundo os autores isto oferece uma promessa para a utilização destes compostos em optoeletrônica.

As ciclodextrinas

(CD) são oligossacarídeos cíclicos, constituído por unidades de glicose (D-(+)-glucopiranose) unidas através de ligações glicosídicas $(\alpha 1,4)$. Schardinger (1903) identificou as ciclodextrinas como produtos resultantes da degradação do amido pela ação da enzima amilase ciclodextrina glicosil transferase (CGTase), produzida pelo microorganismo Bacillus macerans. Este é capaz de romper um segmento da hélice do amido e unir as duas porções terminais deste fragmento numa única molécula cíclica (SAENGER,1980). Por modificação enzimática é possível obter a ciclização de seis, sete ou oito unidades de glicose dando origem à $\alpha, \beta$ e $y-C D$, respectivamente.

Do ponto de vista estrutural, as CD's apresentam-se na forma de cones truncados. Os diâmetros das cavidades da face mais estreita e da mais larga são respectivamente 4,7 e $5,3 \AA$ para uma $\alpha$-CD, 6,0 e $6,5 \AA$ para a $\beta-C D$ e 7,5 e $8,3 \AA$ para $\gamma-C D$.

A presença das hidroxilas livres na parte externa das CD's confere a essas moléculas um caráter hidrofílico. Esse arranjo estrutural das moléculas de glicose nas CD's possibilita a formação de complexos de inclusão com uma variedade de espécies hidrofóbicas ou anfifílicas de tamanho adequado, admitindo-as, pelo menos parcialmente dentro da cavidade, permitindo assim a "dissolução" em meio aquoso de compostos que apresentam baixa solubilidade neste solvente (KURKOV \& LOFTSSON, 2013).

O espaço restrito e a relativamente reduzida polaridade da cavidade da CD podem influenciar às propriedades das moléculas hóspedes incluídas na cavidade.

As propriedades fotofísicas, como intensidade de absorção/emissão e deslocamento no máximo absorção/emissão dos hóspedes, são notavelmente alteradas mediante a complexação com CD.

Krishnamoorty \& Dogra (1999), estudando o efeito da presença de b-CD sobre o comportamento fotofísico da 2-(4'-N,Ndimetilaminofenil)benzimidazola (DMAPBI), observaram o efeito hipercrômico, ou seja, o aumento da intensidade de absorção, da banda mais deslocada para maiores comprimentos de onda, com o aumento da concentração de b-CD, assim como um deslocamento batocrômico de 328 para $333 \mathrm{~nm}$, desta mesma banda associado ao aumento da concentração de b-CD no meio. Os autores justificam este aumento da absorbância devido ao encapsulamento da DMAPBI na cavidade da b-CD.

Estas propriedades têm sido utilizadas nos estudos de equilíbrios de complexação de inclusão entre CD e fluoróforos e a dinâmica do fluoróforo na cavidade CD.

Devido a essas características, CD e seus derivados têm sido amplamente utilizados para estudos fotoquímicos e fotofísicos de estruturas supramoleculares, (REICHARDT, 1988). Geralmente CDs nativas absorvem abaixo de $300 \mathrm{~nm}$, porém, quando grandes cromóforos aromáticos estão ligados a CD ou inclusos nesta, o sistema exibe significante alteração do comportamento espectral. Assim a medição espectrofotométrica torna-se não só um método conveniente, mas também eficiente para investigar o comportamento de complexação de sistemas CD (REICHARDT, 1988).

O termo solvatocromismo descreve as mudanças relevantes nas características (posição, intensidade e forma) de bandas de absorção e/ou emissão eletrônica com a variação da polaridade do meio. $O$ solvatocromismo negativo é definido pelo deslocamento hipsocrômico do Amáx de uma banda de absorção/emissão eletrônica com o aumento da polaridade do solvente. Já o deslocamento batocrômico define 0 solvatocromismo positivo.

PERIÓDICO TCHÊ QUÍMICA • www.periodico.tchequimica.com • Vol. 12 N. 23 - ISSN 1806-0374 (impresso) • ISSN 1806-9827 (CD-ROM) • ISSN 2179-0302 (meio eletrônico) 
O solvatocromismo é produzido pela diferença na solvatação do estado fundamental e do primeiro estado excitado da molécula que absorve a luz (ou o seu cromóforo). Além de importantes para descrever as energias relativas dos estados eletrônicos de moléculas, as mudanças solvatocrômicas são úteis também para a determinação experimental de algumas propriedades físicas importantes, tais como os momentos de dipolo, a polarizabilidade e os momentos de multipolo de moléculas. Deste modo, os efeitos do solvente sobre o espectro de absorção eletrônica e de emissão podem ser usados para fornecer informações acerca das interações soluto-solvente (CARDOSO, 2002).

Com interesse de estudar as propriedades fotofísicas, a fotoestabilidade assim como também aumentar a solubilidade desses derivados azalactônicos em solução aquosa, pensou-se em trabalhar com estruturas supramoleculares que possuam nanocavidades, visando a utilização destes derivados como corantes para lasers ou sensores fluorescentes do ambiente.

Neste trabalho foi estudado o comportamento fotofísico e da fotoestabilidade de um derivado azalactônico em solução de npropanol $(\mathrm{nPrOH})$ e misturas de hidroalcoolicas de n-PrOH/água na presença ou não de b-CD.

\section{MATERIAL E MÉTODOS}

O derivado azalactônico utilizado neste trabalho foi o 4-[4-(dimetilamino)benzilideno]-2fenil-1,3-oxazol-5(4H)-ona, daqui para frente denominado somente como AZA2 (Figura 3). Este derivado foi sintetizado em laboratório pelo método clássico de Plöchl-Erlenmeyer e sua síntese está descrita na literatura (ROSA \& SAVARIZ, 2005).

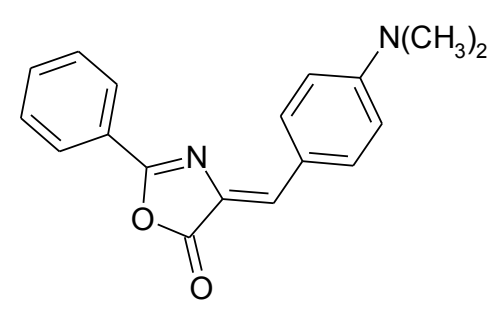

Figura 3 - Estrutura da AZA2
As soluções-mãe e de análise foram preparadas utilizando-se água destilada, npropanol (nPrOH) e N,N-dimetilformamida (DMF), ambos grau P.A., sem qualquer tipo de tratamento.

A b-CD (99\%) foi fornecida pela Aldrich e também foi empregada sem qualquer tipo de purificação.

A preparação da solução-mãe de AZA2 em $\mathrm{nPrOH}$ foi realizada solubilizando-se 0,0290 g $(0,1 \mathrm{mmol})$ de AZA2 em $3 \mathrm{~mL}$ de DMF e completando-se o volume do balão para $100 \mathrm{~mL}$ com $\mathrm{nPrOH}$. A solução foi submetida ao ultrassom por 30 min para completa solubilização e degasagem (retirada do oxigênio molecular), obtendo-se uma solução de concentração de $1 \times 10^{-3} \mathrm{~mol} \mathrm{~L}^{-1}$.

A solução mãe de b-CD foi preparada solubilizando-se $0,283 \mathrm{~g}\left(2,5 \times 10^{-4} \mathrm{~mol}\right)$ em $50 \mathrm{~mL}$ de água destilada obtendo-se uma solução de concentração final $5,0 \times 10^{-3} \mathrm{~mol} \mathrm{~L}^{-1}$.

Para o preparo das amostras sem a presença de b-CD foram utilizados cinco balões de $10 \mathrm{~mL}$ para os quais foram transferidas alíquotas de $0,1 \mathrm{~mL}$ da solução-mãe de AZA2/nPrOH adicionando-se em seguida 1,2 , $3,4,5$ e $7 \mathrm{~mL}$ de água e completando-se o volume com $\mathrm{nPrOH}$.

Para outros cinco balões de $10 \mathrm{~mL}$ foram transferidos alíquotas de $0,1 \mathrm{~mL}$ da soluçãomãe de AZA2/nPrOH adicionando-se em seguida 1, 3, 5 e $7 \mathrm{~mL}$ da solução-mãe de b$\mathrm{CD} / \mathrm{H} 2 \mathrm{O}$ para cada um dos respectivos balões, sendo então o volume completado com $\mathrm{nPrOH}$. A concentração de AZA2 em todos os balões foi de $1 \times 10^{-5} \mathrm{~mol} \mathrm{~L}^{-1}$ e as concentrações de b-CD variaram de 0,5 a $3,5 \times 10^{-3} \mathrm{~mol} \mathrm{~L}^{-1}$.

Todas as soluções preparadas foram submetidas ao ultrassom por 15 min para completa solubilização e retirada de $\mathrm{O} 2$ da solução. Em seguida foi realizado o estudo fotodegradativo pela irradiação em fotorreator (Rayonet RPR-100 - Southern New England Co) equipado com oito (8) lâmpadas com máximo de emissão em 300 nm.

A evolução da degradação foi acompanhada pela variação espectral da amostra verificada pelos espectros de absorção 
obtidos em um espectrofotômetro de varredura UV-vis Shimadzu 1601-PC. Os espectros foram traçados e tratados matematicamente em planilha eletrônica (Excel).

\section{RESULTADOS E DISCUSSÃO:}

A Figura 4 mostra o espectro de absorção da AZA2 em solução de nPrOH. Nesta pode ser observado a presença de uma banda de baixa intensidade em torno de $307 \mathrm{~nm}$ e outra mais intensa em torno de $470 \mathrm{~nm}$. A primeira banda apresenta-se muito pouco estruturada e a segunda sem qualquer tipo de estruturação.

Os espectros de absorção da AZA2 obtidos em solventes de diferentes polaridades apresentaram comportamento solvatôcromico, como pode ser observado na Figura 5 abaixo, na qual o derivado AZA2 sofreu deslocamento batocrômico de cerca de $70 \mathrm{~nm}$ quando a polaridade do solvente foi aumentada.

Nas soluções de AZA2 em nPrOH contendo quantidades crescentes de água, o principal efeito observado foi o deslocamento batocrômico da banda mais deslocada para o vermelho (Figura 6). No detalhe desta Figura é apresentado o gráfico de $\Delta \lambda$ vs $\% \mathrm{H}_{2} \mathrm{O}$ no qual possível se observar que este deslocamento ocorre linearmente ao aumento da quantidade de água no meio.

$A$ adição de $b-C D$ tem como efeito no espectro de UV-vis da AZA2 o deslocamento batocrômico da banda na região do visível proporcionalmente ao aumento da concentração de b-CD no meio, como mostrado na Figura 7. Esse deslocamento pode evidenciar a ocorrência do encapsulamento da AZA2 na cavidade da b-CD, como sugerem os trabalhos encontrados na literatura para outras moléculas. Entretanto, como neste caso a concentração de água também está aumentando, este deslocamento também pode estar associado ao aumento da polaridade do meio, visto que este mesmo comportamento foi observado no experimento que não continha $b-C D$.

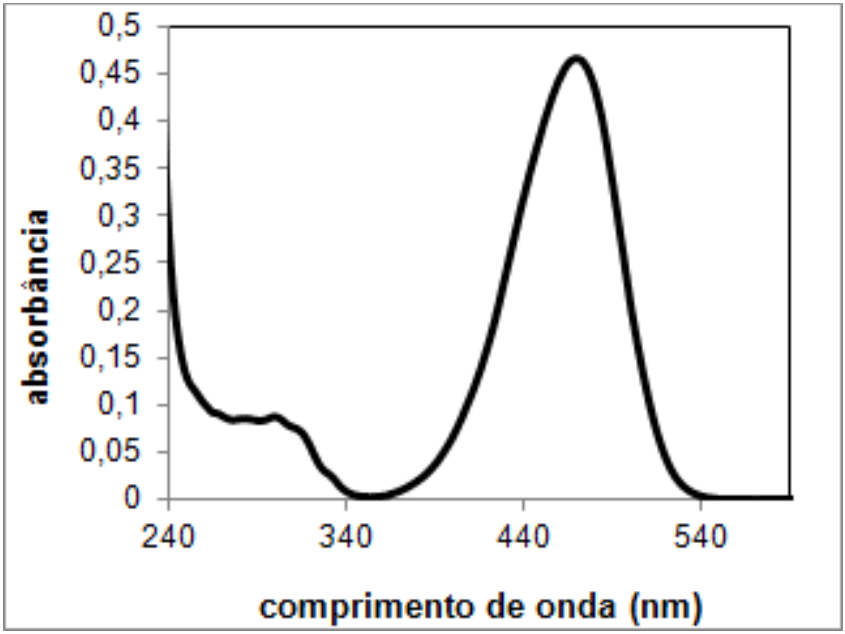

Figura 4 - Espectro de absorção da AZA2 em $n \mathrm{PrOH}$

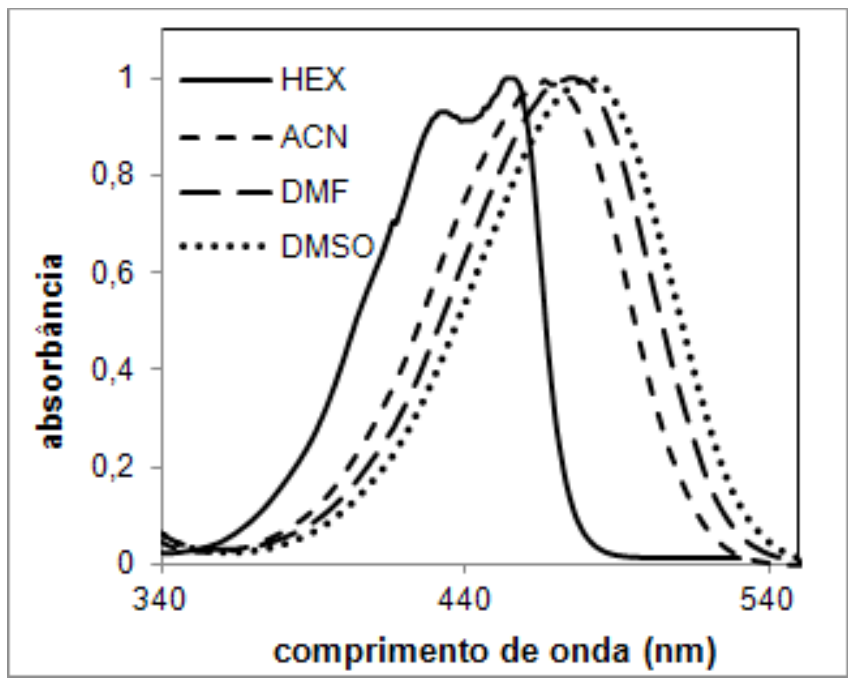

Figura 5 - Espectros de absorção da AZA2 em diferentes solventes nos quais são (HEX) hexano, (ACN) acetonitrila, (DMF) dimetilformamida, (DMSO) dimetilsulfóxido.

Na Figura 8 são mostradas as bandas de absorção obtidas na amostra contendo 30\% água e $30 \%$ água $+b-C D$. Verifica-se que, para esta proporção de água, a adição de b-CD não resultou no deslocamento batocrômico de $\lambda_{\max }$, porém observa-se um pronunciado efeito hipercrômico.

Este comportamento é idêntico aquele observado por Rajendiram \& Balasubramanian (2007), e pode ser explicado pela maior solubilização do derivado azalactônico no meio, o que nos sugere que está ocorrendo encapsulamento da AZA2 na cavidade da b-CD. 
$\mathrm{O}$ estudo da fotoestabilidade da AZA2 em solução de $\mathrm{nPrOH}$ e nas misturas $\mathrm{nPrOH} / \mathrm{H}_{2} \mathrm{O}$ e $\mathrm{nPrOH} / \mathrm{H}_{2} \mathrm{O} / \mathrm{b}-\mathrm{CD}$ foi realizado submetendo-se as amostras à irradiação, e acompanhando-se a degradação por meio dos espectros de absorção obtidos em intervalos de $15 \mathrm{~min}$.

O comportamento observado em todos os experimentos foi idêntico, ou seja, a diminuição da intensidade da banda mais deslocada para o vermelho (banda $\pi \pi^{*}$ ) ao longo do tempo, como mostrado na Figura 9 para um dos experimentos

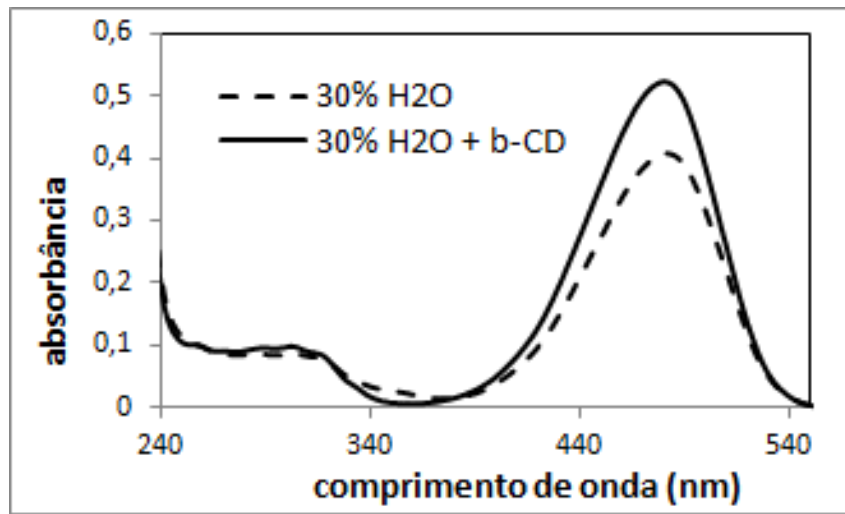

Figura 8 - Espectro de absorção da AZA2 $\left(1 \times 10^{-5} \mathrm{~mol} \mathrm{~L}^{-1}\right)$ em água com e sem b-CD $\left(1,5 \times 10^{-3} \mathrm{~mol} \mathrm{~L}^{-1}\right)$

O estudo cinético da degradação da AZA2 em meio alcoólico e hidroalcoólico foi realizado submetendo-se a irradiação as amostras com diferentes proporções de água e adquirindo-se seus espectros de absorção em intervalos regulares de tempo. Com os valores do máximo de absorção foram traçados os gráficos de $\ln (10 / I)$ vs. tempo para os primeiros 75 minutos de irradiação, intervalo este que apresentou comportamento relativamente linear para todos os experimentos.

A Tabela 1 apresenta os valores de $k$ (constantes de velocidades relativas) obtidos pelo ajuste linear dos pontos para cada um dos experimentos com adição de $\mathrm{H}_{2} \mathrm{O}$.
Tabela 1 - valores das constantes de velocidade calculadas para os processos de fotodegradação em função da percentagem de água.

\begin{tabular}{c|c|c}
\hline $\mathbf{\% H}_{\mathbf{2}} \mathbf{O}$ & $\left.\mathbf{k ~ x ~ 1 \mathbf { 1 0 } ^ { - 3 }} \mathbf{( m i n}^{-\mathbf{1}}\right)$ & $\mathbf{R}^{\mathbf{2}}$ \\
\hline 0 & 8,2 & 0,9801 \\
\hline 10 & 3,4 & 0,9937 \\
\hline 20 & 2,5 & 0,9976 \\
\hline 30 & 2,3 & 0,9993 \\
\hline 40 & 4,3 & 0,9955 \\
\hline 50 & 5,4 & 0,9712 \\
\hline 70 & 6,6 & 0,9812 \\
\hline
\end{tabular}

Observou-se que o processo que apresentou maior constante de velocidade foi aquele que teve somente $\mathrm{o} \mathrm{nPrOH}$ como solvente $\left(k=8,2 \times 10^{-3} \mathrm{~min}^{-1}\right)$. Este valor foi cerca de quatro vezes superior do que aquele obtido para o processo com $30 \%$ de água $(\mathrm{k}=$ $2,3 \times 10^{-3} \min ^{-1}$ ).

Inicialmente este resultado não surpreende, visto que estudos anteriores (ROSA \& SAVARIZ, 2005) mostram que a presença de solventes doadores de hidrogênio, como o iPrOH, aceleram de forma significativa a fotodegradação de estruturas azalactônicas. Desta forma o $\mathrm{nPrOH}$ deve estar atuando como doador de hidrogênio como visto em estudos anteriores.

Para o experimento realizado com $30 \%$ de água esperar-se-ia que a presença desta iria atuar como doador de hidrogênio acelerando degradação do derivado azalactônico, o que de fato não acontece. Uma explicação para tal comportamento pode ser a solvatação preferencial. Como o derivado azalactônico possui baixa solubilidade em água, este deve estar interagindo principalmente com a porção alifática da estrutura do álcool, enquanto a porção polar deste interage com a água, diminuido assim a transferência de hidrogênio.

Decidiu-se então realizar 0 processo fotodegradativo da AZA2 na presença de b-CD somente para o experimento que continha $30 \%$ de água no meio $([b-C D]=1,5 \times 10-3 \mathrm{~mol} L-1)$. $O$ valor de $k$ calculado pelo método gráfico $(R 2=$ 0,9947 ) foi de $3,7 \times 10-3 \mathrm{~min}-1$, ou seja, aproximadamente o dobro daquele obtido para o processo sem a presença de b-CD. Este resultado obtido foi surpreendente, pois 
esperava-se que, com o encapsulamento, houvesse um aumento na estabilidade do corante.

Entretanto há alguns relatos na literatura sobre este tipo de comportamento. Wang et al. (2007) investigaram o comportamento de fotodegradação do bis-(4-hidroxifenil)etano (BPE) na presença de $\alpha-, \beta-$ e $\gamma-C D$. Os autores observaram que a presença de $Y-C D$ confere uma excelente estabilização à molécula quando exposta a irradiação UV. Entretanto o comportamento oposto é observado na presença de b-CD.

Segundo os autores o processo de fotooxidação do BPE é dependente da profundidade de inclusão. Quando na presença de $\mathrm{Y}-\mathrm{CD}$ a molécula está totalmente incluída na cavidade não sofrendo degradação apreciável, pois se encontra afastada dos grupos hidroxilas secundários da molécula de $\mathrm{y}-\mathrm{CD}$. Entretanto na presença de b-CD o BPE não está totalmente incluso na cavidade possuindo proximidade suficiente aos grupos hidroxilas secundárias da cavidade. Estes grupos podem ser ativados, convertendo-se em radicais hidroxila sob irradiação UV, aumentando assim a oxidação fotoquímica.

Assim, uma explicação para a b-CD atuar como agente de degradação seria a ocorrência da inserção parcial da AZA2 na cavidade, ficando parte da estrutura próxima as hidroxilas secundárias na cavidade da b-CD, acelerando o processo de degradação.

\section{CONCLUSÕES:}

O derivado AZA2 apresentou comportamento solvatocrômico. Foi observado que ocorre encapsulamento da AZA2 na cavidade da b-CD quando em solução hidroalcoolica, que foi evidenciado pelo efeito hipercrômico da banda absorção em meio contendo água e b-CD.

Quanto à fotoestabilidade verifica-se uma maior fotoestabilidade do corante em meio hidroalcoolico, provavelmente devido à solvatação preferencial. Em relação ao complexo AZA2@b-CD verifica-se que a b-CD atua como agente de degradação em meio hidroalcoólico, diminuindo, assim, a fotoestabilidade da AZA2 quando encapsulada em b-CD. Este comportamento pode ser explicado pela inclusão parcial da molécula hóspede na cavidade do hospedeiro.

\section{REFERÊNCIAS:}

1. CARDOSO, D. S. Investigação das interações soluto-solvente e solventesolvente na solvatação preferencial da merocianina de Brooker em misturas binárias de solventes. Florianópolis 2002, 4-16. Dissertação (Mestrado em Química) - Universidade Federal de Santa Catarina.

2. ERLENMEYER, F. Ueber die Condensation der Hippursäure mit Phtalsäureanhydrid und mit Benzaldehyd, Justus Lieb Ann. Chem. 1892, 271: 137.

3. ERTEKIN, K.; ALP, S; KARPIRE, C.; YENIGÜL, B.; HENDEN, E.; IÇLI, S. Fluorescence emission studies of an azlactone derivative embedded in polymer films: An optical sensor for $\mathrm{pH}$ measurements J. Photochem. Photobiol. A 2000, 137: 155-161.

4. ERTEKIN, K.; ALP, S.; YALCIN, I. Photophysical and photochemical characteristics of an azlactone dye in solgel matrix; a new fluorescent $\mathrm{pH}$ indicator. Dyes \& Pigm. 2003, 56[2]: 125133.

5. ERTEKIN, K.; CINAR, S.; AYDEMIR, T.; ALP, S. Glucose sensing employing fluorescent $\mathrm{pH}$ indicator: $4-[(p-N, N-$ dimethylamino)benzylidene]-2-

phenyloxazole-5-one Dyes \& Pigm. 2005, 67[2]: 133-138.

6. FISK, J. S.; MOSEY, R. A.; TEPE, J. The diverse chemistry of oxazol-5-(4H)-ones Chem. Soc. Rev. 2007, 36: 1432-1440.

7. KITAZAWA. M., HIGUCHI, R.; TAKAHASHI, M.; WADA, T.; SASABE, $H$. Novel molecular design for second harmonic generation: Azlactones derivatives J. Phys. Chem. 1995, 99: 14784-14792.

8. KÓCZÁN, G., et al., Synthesis and characterization of 4-etoxymethylene-2- 
[1]-naphthyl-5(4H)-oxazolone and its fluorescent amino acid derivatives, Tetrahedron, 2001, 57[21]: 4589-4598.

9. KRISHNAMOORTHY, G.; DOGRA, S. K. Dual fluorescence of 2-(4'-N,Ndimethylaminophenyl)benzimidazole:

effect of $\beta$-cyclodextrin and $\mathrm{pH}, J$. Photochem. Photobiol. A 1999, 123: 109119.

10. KURKOV, S.V.; LOFTSSON, T. Cyclodextrins, Int. J. Pharm. 2013, 453, 167-180

11. OZTURK G.; ALP, S.; ERTEKIN, K. Determination of pKa values of azlactone dyes in non- aqueous media, Dyes \& Pigm. 2005, 65: 33-38.

12. OZTURK, G.; ALP, S.; TIMUR, S., Photophysical characterization of fluorescent oxazol-5- one derivatives in PVC and their application as biosensors in the detection of Ach and AChE inhibitor: Donepezil, Dyes \& Pigm. 2008, 76: 792-798.

13. PLOCHL, J. Über einige derivate der benzoylimdozimsäure, Chem. Ber., 1884, 16: 2815 .

14. RAJENDIRAN,

N.;

BALASUBRAMANIAN, T. Dual fluorescence of $N$-phenylanthranilic acid: effect of solvents, $\mathrm{pH}$ and $\beta$-cyclodextrin, Spectrochim. Acta 2007, 68: 867-876.

15. REICHARDT, C. Solvents and Solvent
Effects in Organic Chemistry; VCH: Weinheim; p. 329-387; 1988.

16. ROSA, M.F.; SAVARIZ, F. C. Síntese e estudo da fotoestabilidade de derivados azalactônicos encapsulados em $\beta$ ciclodextrina, Ecl. Quim. 2005, 30[4]: 7581.

17. SAENGER, W. Cyclodextrin inclusioncompounds in research and industry, Angew. Chem. Int. Eng .Ed. 1980, 19: 344-362.

18. SCHARDINGER, F. Über thermophile Bakterien aus verschiendenen Speisen und Milch, sowie über einige Umsetzungprodukte derselben in kohlenhydrathaltigen Nähr1ösungen darunter krystallisierte Polysaccharide (Dextrine) aus Stärke, Z. Untersuch Nahr. u. Genussm. 1903, ㅁ: 865-880.

19. SMOKAL, V.; CZAPLICKI, R.; DERKOWSKA, B.; KRUPKA, O.; KOLENDO, A.; SAHRAOUI Synthesis and study of nonlinear properties of oxazolone containig polymers, Synth. Metals 2007, 157: 708-712.

20. WANG, G.; XUE, X.; LI, H.; WU, F.; DENG, $\quad N$. $\quad \beta$-Cyclodextrin-enhanced photodegradation of bis(4hydroxyphenyl)ethane under UV irradiation,. J. Mol. Cat. A 2007, 276 : 143-149. 


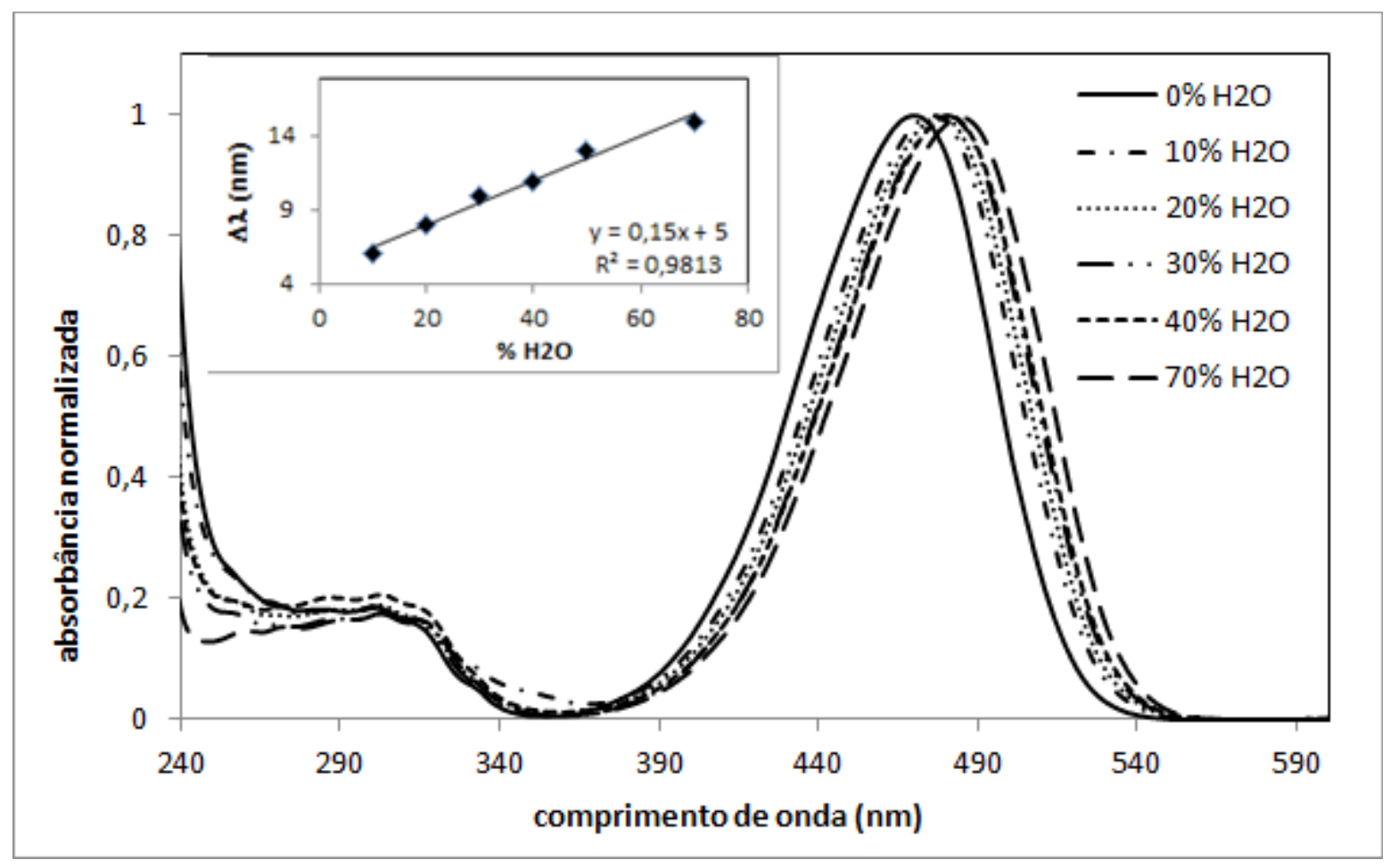

Figura 6 - Espectros de absorção normalizados da AZA2 na banda em $470 \mathrm{~nm}$ em função da quantidade de água na solução.

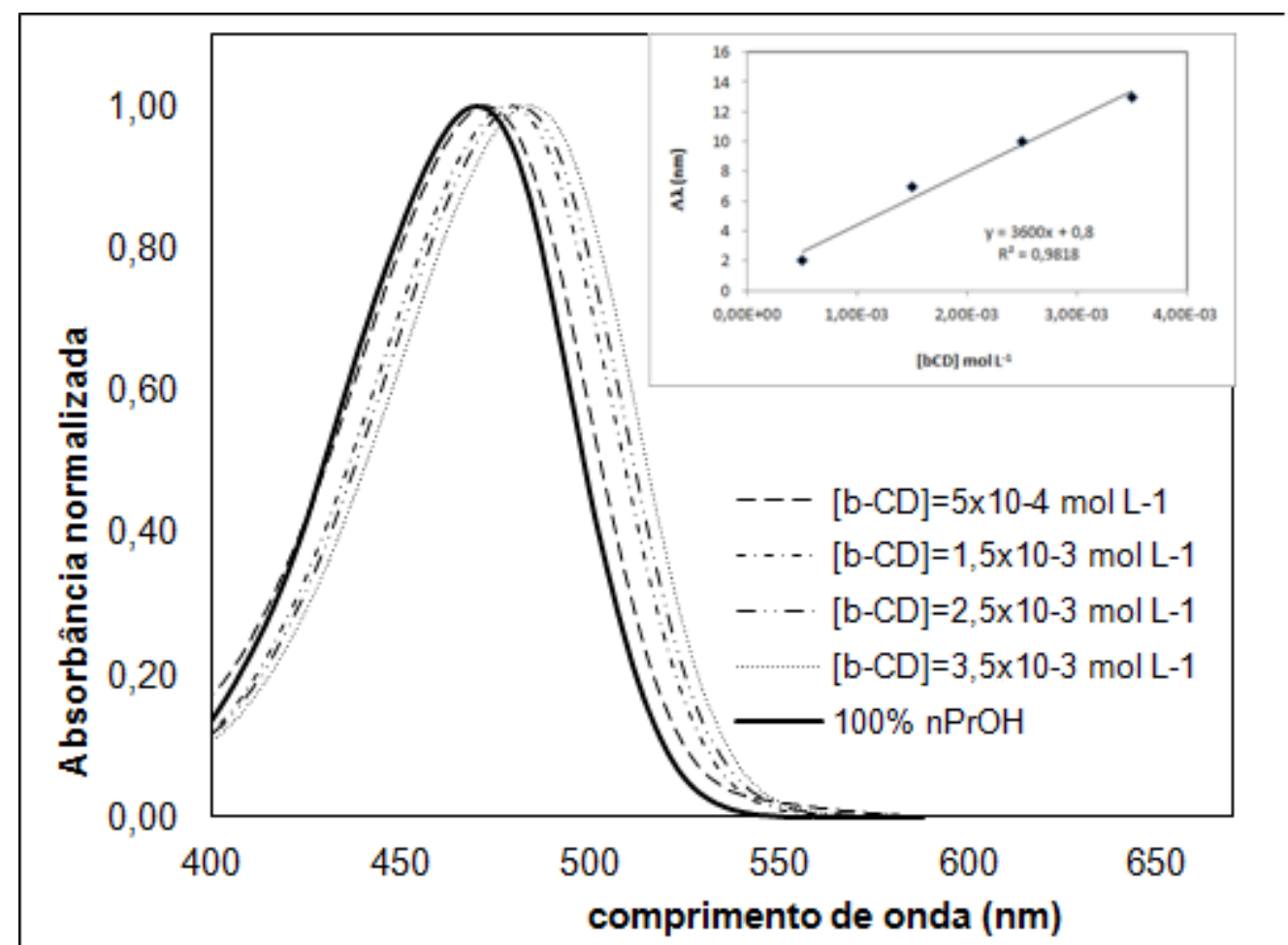

Figura 7 - Espectros de absorção normalizados da AZA2 (banda em $470 \mathrm{~nm}$ ) em função da [b$\mathrm{CD}]$. Os valores [b-CD] encontram-se no gráfico.

PERIÓDICO TCHÊ QUÍMICA • www.periodico.tchequimica.com • Vol. 12 N. 23

- ISSN 1806-0374 (impresso) • ISSN 1806-9827 (CD-ROM) • ISSN 2179-0302 (meio eletrônico)

(C) 2015. Porto Alegre, RS. Brasil 


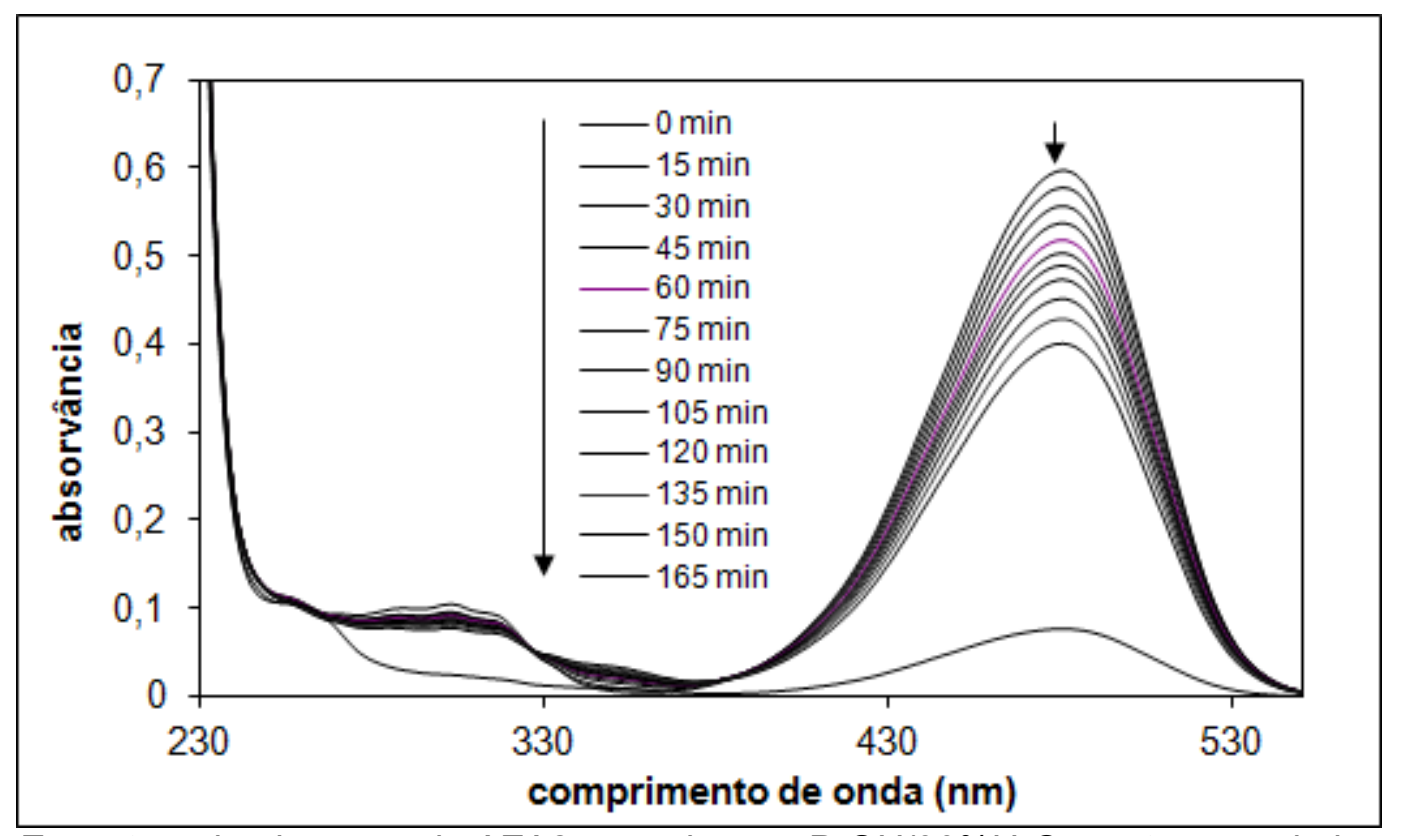

Figura 9 - Espectros de absorção da AZA2 em solução $\mathrm{nPrOH} / 30 \% \mathrm{H}_{2} \mathrm{O}$ nos tempos de irradiação.

PERIÓDICO TCHÊ QUÍMICA • www.periodico.tchequimica.com • Vol. 12 N. 23.

•ISSN 1806-0374 (impresso) • ISSN 1806-9827 (CD-ROM) • ISSN 2179-0302 (meio eletrônico)

$$
\text { (C) 2015. Porto Alegre, RS. Brasil }
$$

